\title{
Effect of Sodium-Glucose Cotransporter-2 Inhibitors versus Dipeptidyl Peptidase 4 Inhibitors on Cardiovascular Function in Patients with Type 2 Diabetes Mellitus and Coronary Artery Disease
}

\author{
Sook Jung Lee, Kook Hyung Lee, Hyun Geong Oh, Hye Ji Seo, Soo Jin Jeong, Chong Hwa Kim* \\ Division of Endocrinology and Metabolism, Department of Internal Medicine, Sejong General Hospital, Bucheon, Korea
}

Background: Randomized controlled trials demonstrated lowering risks of cardiovascular events with sodiumglucose cotransporter-2 (SGLT2) inhibitors in patients with type 2 diabetes mellitus (T2DM) and high cardiovascular risk. We analyzed the effects of cardiovascular function on SGLT2 inhibitors compared with dipeptidyl peptidase-4 (DPP4) inhibitors in T2DM with atherosclerotic cardiovascular disease (ASCVD) or heart failure (HF).

Methods: This is a retrospective, observational, single center study. Data from 89 patients with ASCVD or HF from January 2015 to February 2018 were analyzed regarding the effect of SGLT2 inhibitors and DPP4 inhibitors. Cardiovascular function was assessed by 2-D echocardiography and $\mathrm{N}$-terminal prohormone of brain natriuretic peptide (NT-pro BNP).

Results: A total of 89 patients with T2DM were considered in two groups of SGLT2 inhibitors $(n=41)$ and DPP4 inhibitors $(n=48)$. The mean follow-up period was 2 years, with a total of 89 patient-years. Despite no significant change in systolic function, SGLT2 inhibitors improved cardiovascular function, as demonstrated by a reduced left ventricular ejection fraction less than $40 \%$, ratio of mitral peak velocity of early filling velocity to early diastolic mitral annular velocity, ratio of early to late ventricular filling velocities, and NT-pro BNP compared with the DPP4 inhibitor group.

Conclusion: SGLT2 inhibitors improve cardiovascular function in T2DM with coronary artery disease compared to DPP4 inhibitors.

Key words: Sodium-glucose cotransporter-2, Dipeptidyl peptidase-4 inhibitor, Diabetes Mellitus, Coronary artery disease

\author{
Received October 2, 2019 \\ Reviewed October 31, 2019 \\ Accepted November 28, 2019 \\ ${ }^{*}$ Corresponding author \\ Chong Hwa Kim \\ (iD \\ https://orcid.org/0000-0002-4563-7772 \\ Division of Endocrinology and \\ Metabolism, Department of Internal \\ Medicine, Sejong General Hospital, \\ 28 Hohyeon-ro 489beon-gil, Bucheon \\ 14754, Korea \\ Tel: +82-32-340-1116 \\ Fax: +82-32-340-1236 \\ E-mail: drangelkr@hanmail.net
}

\section{INTRODUCTION}

Prevalence of type 2 diabetes mellitus (T2DM) has been increasing, and T2DM has become a leading cause of cardiovascular mortality in the last decades. ${ }^{1}$ In Korea, patients with T2DM also have higher risk of cardiovascular diseases. ${ }^{2}$ Patients with DM have a higher rate of obesity than non-diabetic patients, indicating that cardiovascular metabolic risk factors may be higher in the T2DM group. ${ }^{2,3}$ Hyperglycemia is significantly related to cardiovascular mortality and morbidity, including myocardial infarction (MI), heart failure (HF), stroke, and hospitalization. ${ }^{4}$ Antihyperglycemic agents have been developed to control hyperglycemia and lower the risk of cardiovascular events. ${ }^{3-5}$ Cardiovascular benefits of sodium-glucose cotransporter-2 (SGLT2) inhibitors have been shown in large-scale randomized trials over the last few years. ${ }^{6-8}$ The Cardiovascular Outcome Trials, including empagliflozin, cardiovascu-

Copyright @ 2019 Korean Society for the Study of Obesity

(a) This is an Open Access article distributed under the terms of the Creative Commons Attribution Non-Commercial License (http://creativecommons.org/licenses/by-nc/4.0/) which permits unrestricted non-commercial use, distribution, and reproduction in any medium, provided the original work is properly cited. 
lar outcomes, and mortality in a type 2 diabetes trial (EMPAREG), Canagliflozin Cardiovascular Assessment Study (CANVAS), and dapagliflozin effect on cardiovascular events-thrombolysis in MI 58, recently demonstrated the benefits of SGLT2 inhibitors in patients with cardiovascular diseases. ${ }^{7,8}$ Some SGLT2 inhibitors have been reported to reduce major adverse cardiovascular events, cardiovascular death, and hospitalization for HF. ${ }^{3,7,8}$ However, the mechanism of SGLT2 inhibitor benefits, including reduction of morbidity, mortality, and $\mathrm{HF}$ aggravation, remains unclear. Regarding cardiovascular diseases, the effects of dipeptidyl peptidase-4 (DPP4) inhibitors were not inferior to those of other antidiabetic drugs. ${ }^{8-10} \mathrm{~A}$ few studies of the effects of SGLT2 inhibitors have been performed in Korea. One report indicated that SGLT2 inhibitors reduced the rate of hospitalization from HF. ${ }^{11}$ For patients with T2DM and coronary artery disease, few studies have been performed to investigate changes in cardiovascular markers with SLGT2 inhibitors compared with DPP4 inhibitors. Discussion is needed regarding differences in cardiovascular function related to SGLT2 inhibitors and DPP4 inhibitors in patients with T2DM and coronary artery disease.

The purpose of this study was to investigate whether SGLT2 inhibitors have positive effects on patients with T2DM and coronary artery disease. SGLT2 inhibitors can induce changes in cardiovascular markers, which may lead to differences between groups treated with SGLT2 inhibitors versus DPP4 inhibitors. Differences between these two groups were investigated by comparing cardiovascular function (systolic blood pressure [BP], diastolic BP, left ven- tricular ejection fraction [LVEF], ratio of mitral peak velocity of early filling velocity to early diastolic mitral annular velocity [E/e'], N-terminal prohormone of brain natriuretic peptide [NT-pro $\mathrm{BNP}]$ ), body weight, body mass index (BMI), random glucose changes, glycosylated hemoglobin (HbAlc), and hospitalization. ${ }^{11}$

\section{METHODS}

\section{Study design}

This study was retrospective and observational. All patients had been diagnosed with established coronary artery diseases (MI, angina). If medications were used (SGLT2 inhibitors, dapagliflozin and empagliflozin vs. DPP4 inhibitors), changes in cardiovascular markers (BP, left ventricular systolic and diastolic function, NT-pro $\mathrm{BNP}), \mathrm{BMI}$, and $\mathrm{HbAlc}$ were measured. This study was approved by Institutional Review Board of Sejong General Hospital (IRB No. 1938). This study was a retrospective chart review with waived patient consent.

A total of 822 patients with T2DM and history of percutaneous coronary intervention (PCI) or coronary artery bypass graft (CABG) were selected from January 2015 to February 2018. CAD was diagnosed by coronary angiography or coronary computed tomography angiography. Intervention or surgery were performed in Sejong General Hospital, Bucheon, Korea. Patients with DM had been taking different anti-diabetic agents (metformin, DPP4 inhibitor, thiazolidinedione, SGLT2 inhibitor, sulfonylurea, and insulin). A total of 444 patients with DM were excluded due to use of other

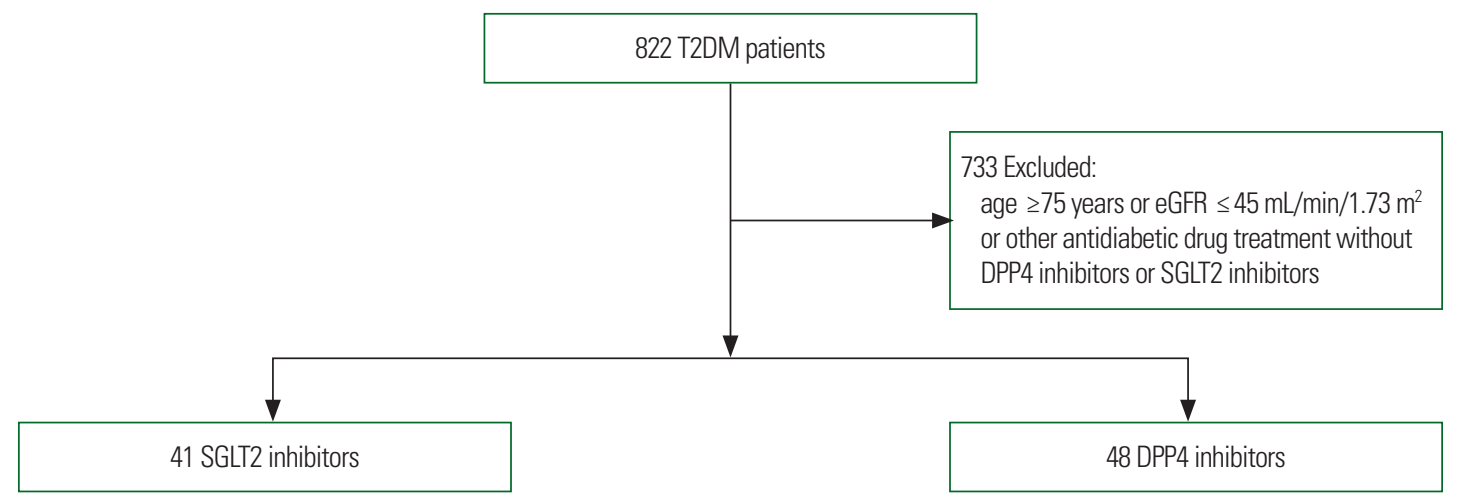

Figure 1. Flowchart of patient selection and grouping. All 822 patients had underwent percutaneous coronary intervention or coronary artery bypass graft and had type 2 diabetes mellitus (T2DM). Four hundred forty-four patients were excluded because they took antidiabetic medications other than sodium-glucose cotransporter-2 (SGLT2) inhibitors or dipeptidyl peptidase-4 (DPP4) inhibitors. Two hundred eighty-nine of 378 patients were excluded for age or estimated glomerular filtration rate (eGFR). Eightynine patients were distributed to SGLT2 inhibitor or DPP4 inhibitor groups. 
antidiabetic agents without DPP4 inhibitors or SGLT2 inhibitors. In summary, 157 patients on SGLT2 inhibitors and patients on 221 DPP4 inhibitors were selected. A total of 289 patients were excluded for age $\geq 75$ years old or estimated glomerular filtration rate $(\mathrm{eGFR}) \leq 45 \mathrm{~mL} / \mathrm{min} / 1.73 \mathrm{~m}^{2}$. Eighty-nine patients with T2DM were identified as new users of SGLT2 inhibitors $(n=41)$ or DPP4 inhibitors $(n=48)$. Patients were divided into two groups: SGLT2 inhibitors and DPP4 inhibitors (Fig. 1).

After PCI or CABG, patients were given SGLT2 inhibitors or DPP4 inhibitors. Echocardiography tests were performed at least twice. The first exam was performed before intervention or CABG. SGLT2 inhibitors or DPP4 inhibitors were prescribed to patients after intervention or surgery. Follow-up echocardiography was performed at least 6 months after initial administration of each drug (SGLT2 inhibitors or DPP4 inhibitors). Changes from the baseline in each group were analyzed. BMI, random glucose level, systolic and diastolic BP, NT-pro BNP, echocardiographic markers (LVEF, E/e', E/A), and hospitalization for HF were assessed. LVEF, E/e', $\mathrm{E} / \mathrm{A}$, and other echocardiographic measures were assessed by Siemens (SC2000; Acuson, Mountain View, CA, USA). Two-dimensional echocardiography was used to evaluate systolic and diastolic cardiac function, such as LVEF, E/e', and E/A. Cardiac structures were shown in two dimensions. LVEF was measured using a biplane method. Doppler was used for E/e'. NT-pro BNP was measured using electrochemiluminescence immunoassay to estimate myocardial stretching status (Roche, Seoul, Korea). Myocardiumderived NT-pro BNP helps to determine cardiac function and HF progression.

Student t-test was performed to analyze baseline characteristics. Multi-response analysis was performed for antidiabetic agents (metformin, sulfonylurea, insulin, DPP4 inhibitors, SGLT2 inhibitors). The SGLT2 inhibitors group and DPP4 inhibitors group were compared by $P$-values. $P$-value $\leq 0.05$ was considered significant. Statistical computations were performed with IBM SPSS version 20.0 (IBM Corp., Armonk, NY, USA).

\section{RESULTS}

The 89 patients were divided into two groups (SGLT2 inhibitors vs DPP4 inhibitors). In the SGLT2 inhibitors group, mean baseline
Table 1. Baseline characteristics of SGLT2 and DPP4 inhibitor groups

\begin{tabular}{|c|c|c|c|}
\hline Variable & $\begin{array}{l}\text { SGLT } 2 \text { inhibitor } \\
\qquad(n=41)\end{array}$ & $\begin{array}{l}\text { DPP4 inhibitor } \\
\quad(n=48)\end{array}$ & $P^{*}$ \\
\hline Age (yr) & $59.98 \pm 8.52$ & $60.96 \pm 6.74$ & 0.542 \\
\hline Male sex & 34 (82.9) & $25(51.0)$ & 0.002 \\
\hline Smoking & & & 0.004 \\
\hline No & $15(45.5)$ & $38(79.2)$ & \\
\hline Yes & $18(54.5)$ & $10(20.8)$ & \\
\hline $\mathrm{PCI} / \mathrm{CABG}$ & & & 0.000 \\
\hline $\mathrm{PCl}$ & 30 (73.2) & $1(2.6)$ & \\
\hline CABG & $9(22.0)$ & $21(53.8)$ & \\
\hline Both & $2(4.9)$ & $17(43.6)$ & \\
\hline Time since diagnosis of T2DM (yr) & $10.09 \pm 8.94$ & $12.95 \pm 7.47$ & 0.132 \\
\hline Weight (kg) & $71.07 \pm 11.04$ & $67.53 \pm 8.24$ & 0.032 \\
\hline Height (cm) & $165.95 \pm 6.89$ & $160.62 \pm 8.24$ & 0.002 \\
\hline $\mathrm{BMI}\left(\mathrm{kg} / \mathrm{m}^{2}\right)$ & $25.80 \pm 0.34$ & $26.17 \pm 2.77$ & 0.855 \\
\hline Systolic blood pressure (mmHg) & $124.88 \pm 14.71$ & $119.28 \pm 16.22$ & 0.061 \\
\hline Diastolic blood pressure (mmHg) & $74.65 \pm 12.57$ & $72.12 \pm 10.86$ & 0.291 \\
\hline $\mathrm{HbA1c}(\%)$ & $7.89 \pm 1.18$ & $7.63 \pm 1.35$ & 0.336 \\
\hline Random glucose (fasting, PP2) (mg/dL) & $198.08 \pm 77.63$ & $156.07 \pm 54.44$ & 0.003 \\
\hline BUN (mg/dL) & $17.34 \pm 5.63$ & $17.84 \pm 6.40$ & 0.707 \\
\hline $\mathrm{Cr}(\mathrm{mg} / \mathrm{dL})$ & $0.93 \pm 0.23$ & $0.98 \pm 0.26$ & 0.316 \\
\hline $\operatorname{eGFR}\left(\mathrm{mL} / \mathrm{min} / 1.73 \mathrm{~m}^{2}\right)$ & $85.68 \pm 16.15$ & $66.84 \pm 16.77$ & 0.000 \\
\hline Hemoglobin (g/dL) & $14.11 \pm 1.66$ & $13.01 \pm 1.55$ & 0.002 \\
\hline NT-pro BNP (pg/mL) & $616.53 \pm 1,155.86$ & $718.53 \pm 708.43$ & 0.769 \\
\hline Ejection fraction (\%) & $46.2 \pm 13.5$ & $56.7 \pm 16.1$ & 0.003 \\
\hline $\mathrm{E} / \mathrm{A}$ & $0.761 \pm 0.322$ & $0.839 \pm 0.349$ & 0.444 \\
\hline $\mathrm{E} / \mathrm{e}^{\prime}$ & $11.37 \pm 4.77$ & $12.87 \pm 5.44$ & 0.380 \\
\hline Hospitalization (day) & $7.76 \pm 10.05$ & $11.16 \pm 11.39$ & 0.149 \\
\hline Antidiabetic agent & SGLT2 inhibitors & DPP4 inhibitors & 0.175 \\
\hline Monotherapy & $2(5.0)$ & $2(4)$ & \\
\hline \multicolumn{4}{|l|}{ Dual combination } \\
\hline Metformin or sulfonylurea & $16(39)$ & $26(54)$ & \\
\hline \multicolumn{4}{|l|}{ Triple combination } \\
\hline Metformin+sulfonylurea & $12(29)$ & $10(21)$ & \\
\hline Metformin+insulin & $4(9.8)$ & $7(15)$ & \\
\hline \multicolumn{4}{|l|}{ Quadruple combination } \\
\hline Metformin+sulfonylurea+insulin & $2(5.0)$ & 2 (4) & \\
\hline Metformin+TZD+insulin & $1(2.4)$ & 0 & \\
\hline Metformin+sulfonylurea+TZD & $4(9.8)$ & 1 (2) & \\
\hline FU period $(\mathrm{mo})$ & & & 0.295 \\
\hline$\leq 6$ & $10(24.4)$ & $4(8.3)$ & \\
\hline$\leq 12$ & $10(24.4)$ & $1(2.1)$ & \\
\hline$\leq 24$ & $9(22)$ & $9(18.8)$ & \\
\hline$>24$ & $12(29.2)$ & $34(70.8)$ & \\
\hline
\end{tabular}

Values are presented as mean \pm standard deviation or number $(\%)$.

${ }^{*} P<0.05$, statistically significant difference.

SGLT2, sodium-glucose cotransporter-2; DPP4, dipeptidyl peptidase-4; PCl, percutaneous coronary intervention; $\mathrm{CABG}$, coronary artery bypass graft; T2DM, type 2 diabetes mellitus; BMl, body mass index; HbA1c, glycated hemoglobin; PP2, post prandial plasma glucose 2 hours; BUN, blood urea nitrogen; $\mathrm{Cr}$, creatinine; eGFR, estimated glomerular filtration rate; NT-pro BNP, N-terminal prohormone of brain natriuretic peptide; E/A, ratio of early $(E)$ to late $(A)$ ventricular filling velocities; $E / e^{\prime}$, ratio of mitral peak velocity of early filling velocity to early diastolic mitral annular velocity; TZD, thiazolidinedione; FU, follow-up. 
age was 59.98 years, diabetes duration was 10.09 years, $\mathrm{HbA1C}$ was $7.89 \%$, eGFR was $815.68 \mathrm{~mL} / \mathrm{min} / 1.73 \mathrm{~m}^{2}$, and BMI was $25.80 \mathrm{~kg} / \mathrm{m}^{2}$. In the DPP4 inhibitors group, mean baseline age was 60.96 years, diabetes duration was 12.95 years, $\mathrm{HbA1C}$ was $7.63 \%$, eGFR was $66.84 \mathrm{~mL} / \mathrm{min} / 1.73 \mathrm{~m}^{2}$, and BMI was $26.17 \mathrm{~kg} / \mathrm{m}^{2}$. At the beginning of this study, there were several significant differences between these two groups. Male patients $(n=34,82.9 \%)$ were dominant in the SGLT2 inhibitor group. Random glucose (198.08 \pm $77.63 \mathrm{mg} / \mathrm{dL}$ ) was higher in the SGLT2 inhibitor group. eGFR $\left(85.68 \pm 16.15 \mathrm{~mL} / \mathrm{min} / 1.73 \mathrm{~m}^{2}\right)$ was also higher in the SGLT2 inhibitor group. Smoking rate was higher in the SGLT2 inhibitor group ( $54.5 \%$ vs. $20.8 \%$ ), and the number of non-smokers was lower in the DPP4 inhibitor group (45.5\% vs. $79.2 \%$ ) (Table 1). Prior to PCI or CABG, there was no significant difference in antidiabetic agent prescription between the SGLT2 inhibitor group and the DPP4 inhibitor group $(P=0.175)$. The follow-up period was not different between the SGLT2 inhibitor group and the DPP4 inhibitor group ( $P=0.295$ for both). However, there was a tendency for a longer follow up period (especially $>24$ months follow $=$ up, 70.8\%) after PCI or CABG in the DPP4 inhibitor group (Table 1). Most patients (in both groups) had taken multiple antidiabetic medications (double or triple or quadruple combination, $39 \%$ vs. $54 \%$ or $38.8 \%$ vs. $36 \%$ or $17.2 \%$ vs. $6 \%$, respectively).

Cardiac function markers, such as NT-pro BNP $(\mathrm{ng} / \mathrm{mL})$ and $\mathrm{BP}$ (systole and diastole, $\mathrm{mmHg}$ ), showed significant changes in the SGLT 2 inhibitor group compared with the DPP4 inhibitor group. Reduced systolic pressure $(5.65 \pm 15.21 \mathrm{mmHg}, P=0.015)$, diastolic pressure $(4.95 \pm 14.58 \mathrm{mmHg}, P=0.049)$, and NT-pro BNP $(374.02 \pm 956.01 \mathrm{ng} / \mathrm{mL}, P=0.034)$ were observed in the SGLT2 inhibitor group (Table 2). No significant difference in LVEF was observed between these two groups (Fig. 2). Subgroup analysis was performed based on degree of LVEF ( $<40 \%, 40 \%-50 \%$, $\geq 50 \%)$ and $\mathrm{E} / \mathrm{e}^{\prime}(<13, \geq 13)$. The SGLT2 inhibitor group had improved LV dysfunction, especially in the reduced LVEF group (LVEF < 40\%; $-9.57 \pm 8.39, P=0.035)$. Diastolic dysfunction markers (E/e', 6.54 \pm 2.95$)$ and NT-pro BNP were also improved in the SGLT2 inhibitor group $(P=0.008)$, especially in the $\mathrm{E} / \mathrm{e}^{\prime}$ $\geq 13$ subgroup (Table 3 ). The DPP4 inhibitor group had a higher rate of rehospitalization ( $22 \%$ vs. $55.1 \%, P=0.002)$. The major cause of hospitalization in both groups was coronary artery disease (data not shown). While patients in the SGLT2 inhibitor group had reduced body weight or BMI, there was no difference in BMI

Table 2. Changes of morphologic, chemical, and cardiovascular function findings before and after treatment with SGLT2 inhibitor and DPP4 inhibitor

\begin{tabular}{|c|c|c|c|c|c|c|c|c|c|}
\hline \multirow{2}{*}{ Variable } & \multirow{2}{*}{$\begin{array}{l}\text { SGLT2 inhibitor } \\
\text { (before) }\end{array}$} & \multirow{2}{*}{$\begin{array}{l}\text { SGLT2 inhibitor } \\
\text { (after) }\end{array}$} & \multirow{2}{*}{$P^{*}$} & \multirow{2}{*}{$\begin{array}{l}\text { DPP4 inhibitor } \\
\text { (before) }\end{array}$} & \multirow{2}{*}{$\begin{array}{l}\text { DPP4 inhibitor } \\
\text { (after) }\end{array}$} & \multirow{2}{*}{$P^{*}$} & \multicolumn{3}{|c|}{ Change in each group } \\
\hline & & & & & & & SGLT 2 inhibitor & DPP4 inhibitor & $P^{*}$ \\
\hline Weight (kg) & $71.07 \pm 11.04$ & $69.69 \pm 10.55$ & 0.558 & $67.53 \pm 8.24$ & $66.40 \pm 9.52$ & 0.424 & $1.47 \pm 4.00$ & $0.97 \pm 5.68$ & 0.662 \\
\hline $\mathrm{BMI}\left(\mathrm{kg} / \mathrm{m}^{2}\right)$ & $25.80 \pm 0.34$ & $25.00 \pm 2.86$ & 0.213 & $26.17 \pm 2.77$ & $25.76 \pm 3.40$ & 0.183 & $0.75 \pm 2.16$ & $0.41 \pm 2.10$ & 0.492 \\
\hline $\begin{array}{l}\text { Systolic blood } \\
\text { pressure (mmHg) }\end{array}$ & $124.88 \pm 14.71$ & $120.03 \pm 12.19$ & 0.129 & $119.28 \pm 16.22$ & $122.12 \pm 13.88$ & 0.110 & $5.65 \pm 15.21$ & $-2.84 \pm 16.74$ & 0.015 \\
\hline $\begin{array}{l}\text { Diastolic blood } \\
\text { pressure (mmHg) }\end{array}$ & $74.65 \pm 12.57$ & $69.70 \pm 9.89$ & 0.438 & $72.12 \pm 10.86$ & $71.61 \pm 10.20$ & 0.616 & $4.95 \pm 14.58$ & $-0.51 \pm 11.26$ & 0.049 \\
\hline $\mathrm{HbA1c}(\%)$ & $7.89 \pm 1.18$ & $7.20 \pm 1.02$ & 0.456 & $7.63 \pm 1.351$ & $6.90 \pm 1.51$ & 0.704 & $0.70 \pm 1.23$ & $0.75 \pm 1.30$ & 0.830 \\
\hline $\begin{array}{l}\text { Random glucose } \\
(\mathrm{mg} / \mathrm{dL})\end{array}$ & $198.08 \pm 77.63$ & $155.57 \pm 52.97$ & 0.003 & $156.07 \pm 54.44$ & $148.67 \pm 63.27$ & 0.608 & $85.42 \pm 97.37$ & $7.39 \pm 75.44$ & 0.000 \\
\hline NT- pro BNP (pg/mL) & $721.59 \pm 1,288.51$ & $346.86 \pm 519.55$ & 0.202 & $561.21 \pm 629.52$ & $661.46 \pm 760.24$ & 0.537 & $374.02 \pm 956.01$ & $-127.812 \pm 755.989$ & 0.034 \\
\hline Ejection fraction (\%) & $46.2 \pm 13.5(n=41)$ & $48.83 \pm 11.57(n=41)$ & 0.273 & $56.7 \pm 16.1(n=40)$ & $56.07 \pm 12.23(n=40)$ & 0.058 & $-2.63 \pm 9.86$ & $0.49 \pm 9.95$ & 0.158 \\
\hline$E / A$ & $0.76 \pm 0.32(n=38)$ & $0.81 \pm 0.31(n=38)$ & 0.839 & $0.84 \pm 0.35(n=19)$ & $0.77 \pm 0.29(n=19)$ & 0.481 & $-0.03 \pm 0.39$ & $-0.05 \pm 0.47$ & 0.088 \\
\hline $\mathrm{E} / \mathrm{e}^{\prime}$ & $11.37 \pm 4.77(n=38)$ & $10.82 \pm 4.12(n=38)$ & 0.420 & $12.87 \pm 5.44(n=21)$ & $10.58 \pm 3.25(n=21)$ & 0.189 & $0.48 \pm 6.32$ & $0.52 \pm 4.59$ & 0.336 \\
\hline \multicolumn{2}{|c|}{ Frailty (rehospitalization) } & & & & & & & & 0.002 \\
\hline \multicolumn{2}{|c|}{ Yes } & & & & & & $9(22.0)$ & $27(55.1)$ & \\
\hline \multicolumn{2}{|l|}{ No } & & & & & & $32(78.0)$ & $22(44.9)$ & \\
\hline
\end{tabular}

Values are presented as mean \pm standard deviation.

${ }^{*} P<0.05$, statistically significant difference.

SGLT2, sodium-glucose cotransporter-2; DPP4, dipeptidyl peptidase-4; BMI, body mass index; HbA1c, glycated hemoglobin; NT-pro BNP, N-terminal prohormone of brain natriuretic peptide; $E / A$, ratio of early (E) to late (A) ventricular filling velocities; E/e', ratio of mitral peak velocity of early filling velocity to early diastolic mitral annular velocity. 


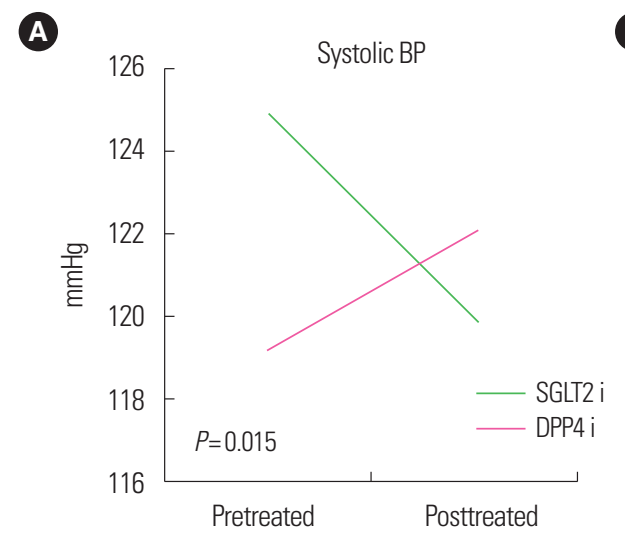

D

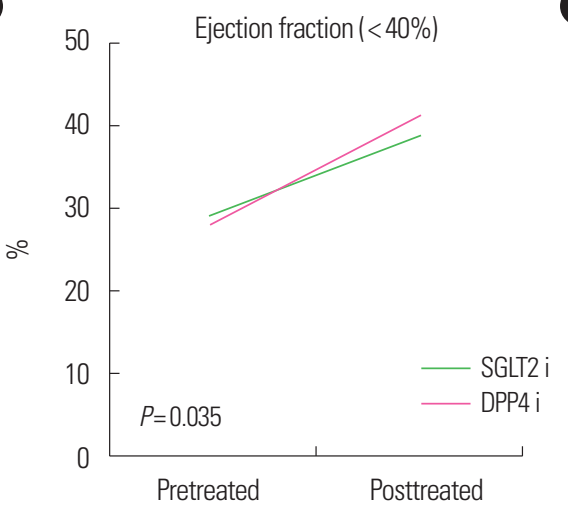

B

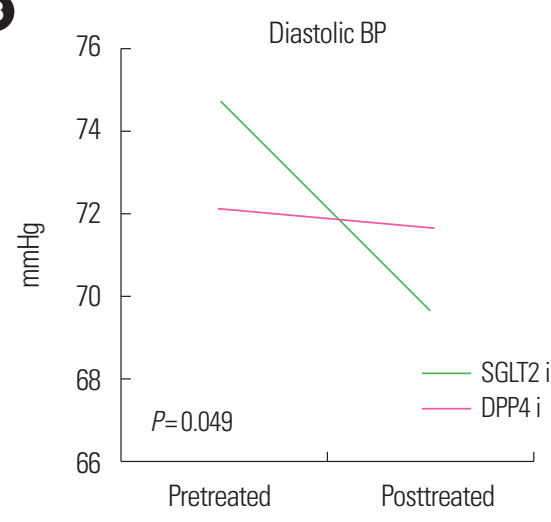

E

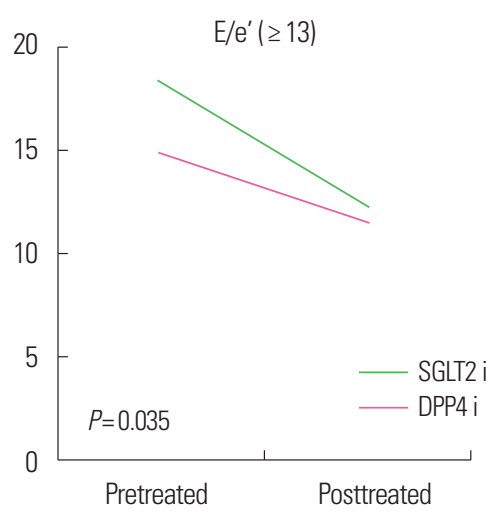

C

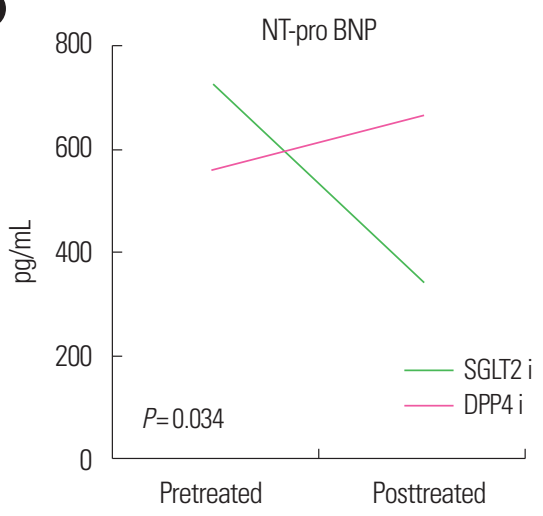

Figure 2. Changes of cardiac markers before and after treatment with sodium-glucose cotransporter-2 inhibitor (SGLT2i) or dipeptidyl peptidase-4 inhibitor (DPP4i). Changes of blood pressure (BP; systole $[A]$, diastole $[\mathrm{B}])$, N-terminal prohormone of brain natriuretic peptide (NTpro BNP [C]), ejection fraction (D), and ratio of mitral peak velocity of early filling velocity to early diastolic mitral annular velocity (E/e' [E]) were significant with SGLT2 inhibitors.

Table 3. Subgroup analysis of cardiac function according to ejection fraction and E/e'

\begin{tabular}{|c|c|c|c|c|c|c|c|c|c|}
\hline \multirow{2}{*}{ Variable } & \multirow{2}{*}{$\begin{array}{l}\text { SGLT2 inhibitor } \\
\text { (before) }\end{array}$} & \multirow{2}{*}{$\begin{array}{l}\text { SGLT2 inhibitor } \\
\text { (after) }\end{array}$} & \multirow{2}{*}{$P^{*}$} & \multirow{2}{*}{$\begin{array}{l}\text { DPP4 inhibitor } \\
\text { (before) }\end{array}$} & \multirow{2}{*}{$\begin{array}{l}\text { DPP4 inhibitor } \\
\text { (after) }\end{array}$} & \multirow{2}{*}{$P^{*}$} & \multicolumn{3}{|c|}{ Change in each group } \\
\hline & & & & & & & SGLT 2 inhibitor & DPP4 inhibitor & $P^{*}$ \\
\hline \multicolumn{10}{|c|}{ Ejection fraction (\%) } \\
\hline$<40$ & $29.00 \pm 6.21(n=13)$ & $38.54 \pm 9.20(n=13)$ & 0.089 & $28.00 \pm 6.60(n=6)$ & $40.83 \pm 10.69(n=6)$ & 0.119 & $-9.57 \pm 8.39(n=14)$ & $-10.5 \pm 13.75(n=6)$ & 0.035 \\
\hline $40-50$ & $45.71 \pm 3.20(n=7)$ & $49.00 \pm 12.46(n=7)$ & 0.030 & $45.80 \pm 2.17(n=5)$ & $50.20 \pm 7.63(n=5)$ & 0.198 & $-2.16 \pm 11.43(n=6)$ & $-4.40 \pm 9.04(n=5)$ & 0.363 \\
\hline$\geq 50$ & $57.00 \pm 4.57(n=21)$ & $55.14 \pm 7.72(n=21)$ & 0.148 & $64.52 \pm 9.68(n=29)$ & $61.10 \pm 9.90(n=29)$ & 0.790 & $1.86 \pm 7.83(n=21)$ & $3.62 \pm 7.47(n=29)$ & 0.925 \\
\hline \multicolumn{10}{|l|}{$\mathrm{E} / \mathrm{e}^{\prime}$} \\
\hline$<13$ & $9.19 \pm 1.78(n=29)$ & $10.44 \pm 4.38(n=29)$ & 0.019 & $10.72 \pm 1.29(n=14)$ & $10.94 \pm 3.85(n=14)$ & 0.013 & $-1.25 \pm 4.64(n=29)$ & $0.33 \pm 3.89(n=15)$ & 0.553 \\
\hline$\geq 13$ & $18.32 \pm 4.65(n=9)$ & $12.27 \pm 2.62(n=9)$ & 0.094 & $14.85 \pm 1.19(n=7)$ & $11.56 \pm 2.43(n=7)$ & 0.073 & $6.54 \pm 2.95(n=8)$ & $8.98 \pm 10.05(n=10)$ & 0.008 \\
\hline
\end{tabular}

Values are presented as mean \pm standard deviation. Systolic function (ejection fraction), especially $<40$ group, improved significantly in SGLT2 inhibitor group ( $P=0.035)$. Diastolic dysfunction (E/e'), especially $\geq 13$ group, improved in SGLT2 inhibitor group ( $P=0.008)$.

${ }^{*} P<0.05$, statistically significant difference.

E/e', ratio of mitral peak velocity of early filling (E) to early diastolic mitral annular velocity (E'); SGLT2, sodium-glucose cotransporter-2; DPP4, dipeptidyl peptidase-4.

between these two groups $\left(0.75 \pm 2.16\right.$ vs. $0.41 \pm 2.10 \mathrm{~kg} / \mathrm{m}^{2}, P=$ 0.492).

\section{DISCUSSION}

This study was designed to assess differences between patients with T2DM and CAD treated with SGLT2 inhibitors or DPP4 inhibitors. After PCI or CABG was performed, SGLT2 inhibitors or
DPP4 inhibitors were prescribed to two different groups. Patient distribution (SGLT2 inhibitors vs DPP4 inhibitors) was similar to that of the EMPA-REG trial. ${ }^{7}$ Our study included patients with CAD but not cerebrovascular diseases. Conversely, the EMPAREG trial included patients with $\mathrm{CAD}$ and cerebrovascular diseases. BP (systole, diastole), NT-pro BNP, systolic cardiac functions (LVEF), and diastolic cardiac function marker $\left(\mathrm{E} / \mathrm{e}^{\prime}\right)$ were significantly reduced, especially in the SGLT2 inhibitors group. No sig- 
nificant difference in ejection fraction was observed between groups. However, for HFrEF (EF < 40\%), LVEF improved in the SGLT2 inhibitors group $(P=0.035)$. The SGLT2 inhibitors group had decreased hospitalization for $\mathrm{CAD}$ and $\mathrm{HF}$ compared with the DPP4 inhibitors group $(P=0.002)$. The EMPA-REG trial showed lower risk of cardiovascular diseases and lower risks of mortality or morbidity, especially hospitalization. ${ }^{9}$ Our study showed that SGLT2 inhibitors reduced the hospitalization rate. The multicenter randomized EMPA-REG trial suggested that empagliflozin reduced the relative risks of HF hospitalization, cardiovascular death, and kidney disease progression rate. ${ }^{7,9}$ The CANVAS trial published that canagliflozin lowered the risk of cardiovascular events and decreased the deterioration rate of renal clearance. ${ }^{12}$ In the CVD-REAL study, the SGLT2 inhibitor group had a lower rate of hospitalization from HF. ${ }^{11}$ SGLT2 inhibitors decreased the risk of both HF hospitalization and mortality. These clinical trials showed that SGLT2 inhibitors have positive effects on patients with cardiovascular diseases. ${ }^{9,12,13}$ DPP4 inhibitors were determined not to be inferior, but saxagliptin was associated with significantly increased rate of HF hospitalization. ${ }^{10}$

This study was designed to evaluate if SGLT2 inhibitors improve cardiac function by assessing several cardiac biomarkers. Reduced systolic and diastolic BP was more obvious in the SGLT2 inhibitors group than in the DPP4 inhibitors group $(P=0.015$ vs. $P=0.049)$. Natriuresis and glycosuria caused by SGLT 2 transporter inhibition resulted in more significant changes in BP (systolic BP, diastolic BP) in the SGLT 2 inhibitors group. ${ }^{8}$ Blockade of SGLT2 transporters induced urine with sodium and water secretion. Volume depletion caused by glycosuria and natriuresis resulted in decreased BP (systole, diastole). ${ }^{13-15}$ The mechanism is not yet fully understood, but the neurohormonal system might be involved via natriuresis caused by SGLT2 transporters, which leads to altered body fluid volume status. ${ }^{14-16}$ The change in NT-pro BNP was remarkable. Although SGLT2 inhibitors were given to patients for a short amount of time, NT-pro BNP decreased more in the SGLT2 inhibitor group than in the DPP4 inhibitor group. Decreased NT-pro BNP indicates decreased cardiac wall stress ${ }^{13}$ and may indicate improved cardiac function in patients with CAD. However, LVEF was not different between the two groups. Verma ${ }^{1}$ explained that SGLT2 inhibitors relieved afterload via natriuresis, which improved cardiac func- tion. Previous trials can explain that there was no improvement in LVEF with SGLT2 inhibitors in our study. For HFrEF, changes in LVEF with SGLT2 inhibitors were more noticeable than with DPP4 inhibitors. ${ }^{13}$ NT-pro BNP level improved significantly in the SGLT2 inhibitor group, which affected cardiac function and ejection fraction, especially in the HFrEF group.

Saxagliptin Assessment of Vascular Outcomes Recorded in Patients with Diabetes Mellitus (SAVOR)-Thrombolysis in Myocardial Infarction (TIMI) 53 suggested that DPP4 inhibitors were cardiovascular (CV) neutral, but increased HF-induced hospitalization rate. ${ }^{10}$ Scirica et al. ${ }^{17}$ reported that saxagliptin increased the rate of hospitalization due to HF. We also found that DPP4 inhibitors had a higher rate of rehospitalization $(P=0.002)$. Treatment with SGLT2 inhibitors might reduce hospitalization rate by decreasing LV stretching and volume depletion. Another Korean trial showed a lower risk of HF hospitalization after 30 days of treatment with SGLT2 inhibitors compared to DPP4 inhibitors. ${ }^{11}$ Thirty days of SGLT2 inhibitors had a positive protective effect on hospitalization for HF in patients with DM and CVD. SGLT2 inhibitors (canagliflozin, dapagliflozin, empagliflozin) have been reported to prevent cardiovascular events such as MI and stoke. ${ }^{18}$

The "thrifty substrate" or "fuel" hypothesis was independently proposed by Ferrannini et al. ${ }^{19}$ and Mudaliar et al. ${ }^{20}$ They both hypothesized that removal of large amounts of glucose from the body and subsequent reduction of the insulin/glucagon ratio associated with empagliflozin treatment may boost lipid mobilization and oxidation in the liver to stimulate ketogenesis. ${ }^{19,20}$ The resulting metabolic condition, characterized by a mildly hyperketonemic state akin to prolonged fasting, leads to avid myocardial uptake of $\beta$-hydroxybutyrate, which competes with fatty acid oxidation. This substrate shift is considered cardioprotective because oxidation of ketone bodies is more metabolically efficient and may act as a "superfuel" for the heart. ${ }^{19}$

No differences in body weight change were observed between the SGLT2 inhibitor group and DPP4 inhibitor group in this study. Although performed at the same center as our study, Cho et al. ${ }^{21}$ reported that SGLT2 inhibitors significantly reduced body weight. This discrepancy might be because patients treated with DPP4 inhibitors underwent improved life style modifications after undergoing surgery or PCI. The discrepancy could also be a limitation of 
this retrospective study.

There are several limitations. First, there were discrepancies in baseline characteristics of the two groups. SGLT2 inhibitors are known to induce ketoacidosis. Because the study was retrospective, it was impossible to know the ketone body level before treatment with SGLT2 inhibitor or DPP4 inhibitor was initiated. Therefore, changes in ketone bodies were not evaluated. Second, this study was performed at a single center with relatively few patients available for analysis. While there were initially 822 enrolled patients, the SGLT2 inhibitor taken group included merely 41 patients and the DPP4 inhibitor group included 48 patients. Few patients had data available for EF (LV systolic function) or E/e' (diastolic function). Third, total observation time (38 months) after launching new drugs such as empagliflozin and dapagliflozin was insufficient to prove potential cardiovascular benefits for patients with coronary diseases.

SGLT2 inhibitors improved BP, hospitalization for CAD or HF, and cardiovascular function (NT-pro BNP, E/e', E/A ratio in the HFrEF group) compared with DPP4 inhibitors. We suggest that SGLT2 inhibitors improve cardiovascular function in patients with T2DM and coronary artery disease compared to DPP4 inhibitors. The cardiovascular effects of SLGT2 inhibitors should be further evaluated in a large scale prospective study in Korea.

\section{CONFLICTS OF INTEREST}

The authors declare no conflict of interest.

\section{AUTHOR CONTRIBUTIONS}

Study concept and design: SJL, KHL, and CHK; acquisition of data: SJL, KHL, HGO, HJS, CHK; analysis and interpretation of data: all authors; drafting of the manuscript: SJL, CHK; critical revision of the manuscript: SJL, SJJ, CHK; statistical analysis: all authors; administrative, technical, or material support: SJJ and CHK; and study supervision: SJJ and CHK.

\section{REFERENCES}

1. Verma S, McMurray JJV. SGLT2 inhibitors and mechanisms of cardiovascular benefit: a state-of-the-art review. Diabetologia
$2018 ; 61: 2108-17$

2. Korean Diabetes Association. Diabetes fact sheet in Korea 2013. Seoul: Korean Diabetes Association; 2013.

3. Cefalu WT, Kaul S, Gerstein HC, Holman RR, Zinman B, Skyler JS, et al. Cardiovascular outcomes trials in type 2 diabetes: where do we go from here? Reflections from a diabetes care editors' expert forum. Diabetes Care 2018;41:14-31.

4. Thompson PL, Davis TME. Cardiovascular effects of glucoselowering therapies for type 2 diabetes: new drugs in perspective. Clin Ther 2017;39:1012-25.

5. Kosiborod M, Lam CS, Kohsaka S, Kim DJ, Karasik A, Shaw J, et al. Cardiovascular events associated with SGLT-2 inhibitors versus other glucose-lowering drugs: the CVD-REAL 2 study. J Am Coll Cardiol 2018;71:2628-39.

6. Neal B, Perkovic V, Mahaffey KW, de Zeeuw D, Fulcher G, Erondu N, et al. Canagliflozin and cardiovascular and renal events in type 2 diabetes. N Engl J Med 2017;377:644-57.

7. Zinman B, Wanner C, Lachin JM, Fitchett D, Bluhmki E, Hantel S, et al. Empagliflozin, cardiovascular outcomes, and mortality in type 2 diabetes. N Engl J Med 2015;373:2117-28.

8. Scheen AJ. Cardiovascular effects of new oral glucose-lowering agents: DPP-4 and SGLT-2 inhibitors. Circ Res 2018;122: 1439-59.

9. Secrest MH, Udell JA, Filion KB. The cardiovascular safety trials of DPP-4 inhibitors, GLP-1 agonists, and SGLT2 inhibitors. Trends Cardiovasc Med 2017;27:194-202.

10. Scirica BM, Bhatt DL, Braunwald E, Steg PG, Davidson J, Hirshberg B, et al. Saxagliptin and cardiovascular outcomes in patients with type 2 diabetes mellitus. N Engl J Med 2013; 369:1317-26.

11. Kim YG, Han SJ, Kim DJ, Lee KW, Kim HJ. Association between sodium glucose co-transporter 2 inhibitors and a reduced risk of heart failure in patients with type 2 diabetes mellitus: a real-world nationwide population-based cohort study. Cardiovasc Diabetol 2018;17:91.

12. Wiviott SD, Raz I, Bonaca MP, Mosenzon O, Kato ET, Cahn A, et al. Dapagliflozin and cardiovascular outcomes in type 2 diabetes. N Engl J Med 2019;380:347-57.

13. Abdelgadir E, Rashid F, Bashier A, Ali R. SGLT-2 inhibitors and cardiovascular protection: lessons and gaps in understand- 
ing the current outcome trials and possible benefits of combining SGLT-2 inhibitors with GLP-1 agonists. J Clin Med Res 2018; 10:615-25.

14. Verma S, Garg A, Yan AT, Gupta AK, Al-Omran M, Sabongui A, et al. Effect of empagliflozin on left ventricular mass and diastolic function in individuals with diabetes: an important clue to the EMPA-REG outcome trial? Diabetes Care 2016;39: e212-3.

15. Natali A, Nesti L, Fabiani I, Calogero E, Di Bello V. Impact of empagliflozin on subclinical left ventricular dysfunctions and on the mechanisms involved in myocardial disease progression in type 2 diabetes: rationale and design of the EMPA-HEART trial. Cardiovasc Diabetol 2017;16:130.

16. Lambers Heerspink HJ, de Zeeuw D, Wie L, Leslie B, List J. Dapagliflozin a glucose-regulating drug with diuretic properties in subjects with type 2 diabetes. Diabetes Obes Metab 2013; 15:853-62.

17. Scirica BM, Braunwald E, Raz I, Cavender MA, Morrow DA, Jarolim $\mathrm{P}$, et al. Heart failure, saxagliptin, and diabetes mellitus: observations from the SAVOR-TIMI 53 randomized trial. Circulation 2014;130:1579-88.

18. Kosiborod M, Birkeland KI, Cavender MA, Fu AZ, Wilding JP, Khunti K, et al. Rates of myocardial infarction and stroke in patients initiating treatment with SGLT2-inhibitors versus other glucose-lowering agents in real-world clinical practice: results from the CVD-REAL study. Diabetes Obes Metab 2018; 20:1983-7.

19. Ferrannini E, Mark M, Mayoux E. CV protection in the EMPAREG OUTCOME trial: a "thrifty substrate" hypothesis. Diabetes Care 2016;39:1108-14.

20. Mudaliar S, Alloju S, Henry RR. Can a shift in fuel energetics explain the beneficial cardiorenal outcomes in the EMPA-REG OUTCOME study? A unifying hypothesis. Diabetes Care 2016; 39:1115-22.

21. Cho HA, Jung YL, Lee YH, Lee YC, Lee JE, Lee SJ, et al. Efficacy of body weight reduction on the SGLT2 inhibitor in people with type 2 diabetes mellitus. J Obes Metab Syndr 2017;26: 107-13. 